\title{
Diagnosing the subsurface buffer on ground surface temperature under the long-term groundwater pumping: An integrated modeling approach
}

\author{
Chen Yang ${ }^{1}$, You-Kuan Zhang ${ }^{1}$, Kun Huang$^{2}$, Zheng Lu $^{3}$, Tingting Zheng ${ }^{4}$, and Xiaofan \\ Yang $^{3}$ \\ ${ }^{1}$ Southern University of Science and Technology \\ ${ }^{2}$ China University of Geosciences \\ ${ }^{3}$ Beijing Normal University \\ ${ }^{4}$ University of Iceland
}

May 5, 2020

\begin{abstract}
Based on the integrated ParFlow.CLM modeling in the Little Washita basin located in the southwestern Oklahoma in the U.S., the long-term effects of groundwater (GW) pumping on ground surface temperature (GST) are studied. Conclusions are obtained based on the simulation results as follows. The subsurface can be conceptualized as a buffer on variations of GST while GW pumping can weaken this buffer causing hotter summer and colder winter. In the long-term pumping, the variations of GST $(\Delta \mathrm{GST})$ present nonlinear warming trend in average by rapidly increasing in the beginning and gradually reaching dynamic equilibrium. This is due to the attainment of a new equilibrium of the GW flow system with a sustainable pumping rate. Whereas, for unsustainable pumping, it is mainly attributed to the fact that the water table depth finally becomes lower than the critical depth range $(1-10 \mathrm{~m})$. Different coupling depths between ParFlow and CLM are tested in the long-term pumping scenarios since it determines the heat capacity of the subsurface buffer which is important on regulating GST. The buffer with deeper coupling depth is more effective on damping the nonlinearity and the amplitude of $\Delta$ GST. In addition, the effects of coupling depth on GST are more prominent when pumping occurs due to the degenerated thermal properties. In other words, the time-scale for GST to response the different coupling depth is greatly shortened under pumping in contrast to natural state. This study diagnosed the subsurface buffer on variations of GST with the role of coupling depth in the integrated modeling under long-term GW pumping. The results are expected to have implications on integrated modeling in GW depletion areas worldwide.
\end{abstract}

\section{Hosted file}

Manuscript_final.docx available at https://authorea.com/users/287446/articles/412198diagnosing-the-subsurface-buffer-on-ground-surface-temperature-under-the-long-termgroundwater-pumping-an-integrated-modeling-approach 